\title{
Probabilistic interpretation of HJB equations by the representation theorem for generators of BSDEs*
}

\author{
Lishun $\mathrm{Xiao}^{\dagger} \quad$ Shengjun Fan ${ }^{\ddagger} \quad$ Dejian Tian $^{\ddagger}$
}

\begin{abstract}
The purpose of this note is to propose a new approach for the probabilistic interpretation of Hamilton-Jacobi-Bellman equations associated with stochastic recursive optimal control problems, utilizing the representation theorem for generators of backward stochastic differential equations. The key idea of our approach for proving this interpretation lies in the identity between solutions and generators given by the representation theorem. Compared with existing methods, our approach seems to be a feasible unified method for different frameworks and be more applicable to general settings. This can also be regarded as a new application of such representation theorem.
\end{abstract}

Keywords: backward stochastic differential equation; recursive optimal control problem; Hamilton-Jacobi-Bellman equation; representation theorem for generator. AMS MSC 2010: 60H10; 35K20; 49L25.

Submitted to ECP on April 24, 2019, final version accepted on March 18, 2020. Supersedes arXiv: 1701.03871.

\section{Introduction}

After the pioneering work on nonlinear backward stochastic differential equations (BSDEs for abbreviated) by Pardoux and Peng [14], the theory of nonlinear BSDEs has been applied to many fields (see El Karoui, Peng and Quenez [7] for details). The stochastic recursive optimal control problem has been identified as an important application of BSDEs in optimal control fields. Peng [19] first interpreted the viscosity solution of a generalized Hamilton-Jacobi-Bellman equation as the value function of a stochastic recursive optimal control problem which is described by a forward-backward stochastic differential equation (FBSDE for short). Peng [20] introduced a notion of backward semigroups to demonstrate a generalized dynamic programming principle (DPP for short) for a stochastic recursive optimal control problem. He also provided a method of approximation of BSDEs' solutions to prove the probabilistic interpretation for HJB

${ }^{*}$ L. Xiao is supported by the Research Initiation Fundation of Xuzhou Medical University (No. D2018002) and the National Natural Science Foundation of China (Nos. 11601509 and 31801957), S. Fan is supported by the Fundamental Research Funds for the Central Universities (No. 2017XKZD11) and D. Tian is supported by the National Natural Science Foundation of China (No. 11601509).

${ }^{\dagger}$ Department of Biostatistics, Xuzhou Medical University, Xuzhou, Jiangsu, 221004, P.R. China. Corresponding author. E-mail: xiaolishun@xzhmu.edu.cn

${ }^{\ddagger}$ School of Mathematics, China University of Mining and Technology, Xuzhou, Jiangsu, 221116, P.R. China. 
equations in the viscosity sense. In this method several BSDEs with different generators and some estimates for solutions are applied to obtain the required variational inequality (see (3.7) or Definition 3.2).

Since then, many researchers began to investigate stochastic recursive optimal control problem induced by FBSDE systems. Buckdahn and Li [4] studied two-player zero-sum stochastic differential games via FBSDEs; Buckdahn and Hu [3] considered a coupled system of HJB equations associated with a stochastic control problem on a Wiener-Poisson space; Li and Wei [12] researched a stochastic recursive optimal control problem whose value function is described by a fully coupled FBSDE system; Li and Tang [11] studied a stochastic recursive optimal control with the state process being reflected in a bounded convex domain; Buckdahn and Nie [5] considered a stochastic exit time optimal control problem. All the previous works manifested the probabilistic interpretation for corresponding HJB equations with Cauchy problems, Dirichlet or Neumann boundary conditions, under Lipschitz frameworks, by Peng's approximation method with some necessary technical modifications. Under a non-Lipschitz setting, $\mathrm{Pu}$ and Zhang [21] proved the probabilistic interpretation for HJB equations by the approximation of a viscosity solution sequence.

Above all, to the best of our knowledge, the existing methods to process the probabilistic interpretation of HJB equations are all based on the approximation of (BSDEs' or PDEs') solutions. In this note, we would like to propose a new and unified approach to treat this probabilistic interpretation utilizing the representation theorem for generators of BSDEs (see Theorem 2.1). This representation theorem was established by Briand, Coquet, $\mathrm{Hu}$, Mémin and Peng [2] and further extended by Jiang [9, 10]. Essentially, the very crucial step of proving the viscosity solution is to claim a variational inequality (see (3.7) or Definition 3.2), the left hand side (without the sup) of which is actually a generator of a BSDE. The novelty of our approach is that the signs of the required generator and the BSDE's solution inherit directly from each other via the identity given by the representation theorem. Moreover, by our approach we can observe that the probabilistic interpretation can be boiled down to the representation problem for generators of a BSDE, provided the DPP holds. So compared with existing methods, the representation theorem approach is more applicable to general frameworks. And this can also be seen as a new application of such representation theorem.

The rest of this paper is organized as follows: Section 2 gives all necessary notations and some elementary results about BSDEs; Section 3 illustrates the probabilistic interpretation for solutions of HJB equations in the viscosity sense adopting the representation theorem for generators of BSDEs, and provides the uniqueness result of viscosity solutions; Section 4 shows some further discussion about the representation theorem approach.

\section{Preliminaries}

Let $T>0$ be a given finite time horizon, $(\Omega, \mathcal{F}, \mathbf{P})$ a probability space carrying a standard $d$-dimensional Brownian motion $\left(B_{t}\right)_{t \geq 0}$ and $\left(\mathcal{F}_{t}\right)_{t \geq 0}$ the natural $\sigma$-algebra filtration generated by $\left(B_{t}\right)_{t \geq 0}$ with $\mathcal{F}_{0}$ containing all $\mathbf{P}$-null sets of $\mathcal{F}$. Postulate that $\mathcal{F}_{T}=\mathcal{F}$ and $\left(\mathcal{F}_{t}\right)_{t \geq 0}$ satisfies the usual conditions. Throughout this note we use $|\cdot|$ and $\langle\cdot, \cdot\rangle$ to denote the Euclidean norm and scalar product, respectively. The Euclidean norm of a matrix $z \in \mathbf{R}^{n \times d}$ will be denoted by $|z|:=\sqrt{\operatorname{Tr}\left(z z^{*}\right)}$, where and hereafter $z^{*}$ represents the transpose of $z$. We denote by $\mathcal{S}^{2}\left(0, T ; \mathbf{R}\right.$ ) (or $\mathcal{S}^{2}$ for brevity) the set of real valued, $\left(\mathcal{F}_{t}\right)$-adapted and continuous processes $\left(y_{t}\right)_{t \in[0, T]}$ such that $\mathbf{E}\left[\sup _{t \in[0, T]}\left|y_{t}\right|^{2}\right]<\infty$. Let $\mathcal{H}^{2}\left(0, T ; \mathbf{R}^{n}\right)$ (or $\mathcal{H}^{2}$ for brevity) denote the set of $\mathbf{R}^{n}$-valued and $\left(\mathcal{F}_{t}\right)$-progressively measurable processes $\left(z_{t}\right)_{t \in[0, T]}$ satisfying that $\mathbf{E}\left[\int_{0}^{T}\left|z_{s}\right|^{2} \mathrm{~d} s\right]<\infty$. 
Next we introduce some elementary results about BSDEs of the following type,

$$
Y_{t}=\xi+\int_{t}^{T} g\left(\omega, s, Y_{s}, Z_{s}\right) \mathrm{d} s-\int_{t}^{T}\left\langle Z_{s}, \mathrm{~d} B_{s}\right\rangle, \quad t \in[0, T] .
$$

If we assume that the terminal data $\xi$ is $\mathcal{F}_{T}$-measurable and $\mathbf{E}|\xi|^{2}<\infty$, the generator $g: \Omega \times[0, T] \times \mathbf{R} \times \mathbf{R}^{d} \mapsto \mathbf{R}$ is $\left(\mathcal{F}_{t}\right)$-progressively measurable and satisfies

(A1) $\{g(\omega, t, 0,0)\}_{t \in[0, T]} \in \mathcal{H}^{2}$;

(A2) There exists a constant $K \geq 0$ such that $\mathrm{d} \mathbf{P} \times \mathrm{d} t-a . e$. , for each $y, y^{\prime} \in \mathbf{R}$ and $z$, $z^{\prime} \in \mathbf{R}^{d}$

$$
\left|g(\omega, t, y, z)-g\left(\omega, t, y^{\prime}, z^{\prime}\right)\right| \leq K\left(\left|y-y^{\prime}\right|+\left|z-z^{\prime}\right|\right),
$$

then by the result of Pardoux and Peng [14] the previous BSDE admits a unique solution $\left(Y_{t}, Z_{t}\right)_{t \in[0, T]}$ in $\mathcal{S}^{2} \times \mathcal{H}^{2}$.

We now proceed to introduce the representation theorem for generators of BSDEs. Assume that the generator $g$ satisfies (A1)-(A2) and fix a triplet $(t, y, z) \in[0, T) \times \mathbf{R} \times \mathbf{R}^{d}$. Then, for each $\varepsilon$ with $0<\varepsilon \leq T-t$, the following BSDE admits a unique solution $\left(Y_{s}^{\varepsilon}, Z_{s}^{\varepsilon}\right)_{s \in[t, t+\varepsilon]}$ in $\mathcal{S}^{2} \times \mathcal{H}^{2}$,

$$
Y_{s}^{\varepsilon}=y+\left\langle z, B_{t+\varepsilon}-B_{t}\right\rangle+\int_{s}^{t+\varepsilon} g\left(\omega, r, Y_{r}^{\varepsilon}, Z_{r}^{\varepsilon}\right) \mathrm{d} r-\int_{s}^{t+\varepsilon}\left\langle Z_{r}^{\varepsilon}, \mathrm{d} B_{r}\right\rangle, \quad s \in[t, t+\varepsilon] .
$$

Theorem 2.1 (Theorem 3.3 in [9] and Lemma 2.1 in [10]). Assume that $g$ satisfies (A1)(A2) and $1 \leq p<2$. Then for each $(t, y, z) \in[0, T) \times \mathbf{R} \times \mathbf{R}^{d}$,

$$
L^{p}-\lim _{\varepsilon \rightarrow 0^{+}} \frac{1}{\varepsilon} \mathbf{E}\left[\left(Y_{t}^{\varepsilon}-y\right)-\int_{t}^{t+\varepsilon} g(\omega, r, y, z) \mathrm{d} r \mid \mathcal{F}_{t}\right]=0
$$

and for each $(y, z) \in \mathbf{R} \times \mathbf{R}^{d}$, the following equality

$$
g(\omega, t, y, z)=L^{p}-\lim _{\varepsilon \rightarrow 0^{+}} \frac{1}{\varepsilon}\left(Y_{t}^{\varepsilon}-y\right)
$$

holds for almost every $t \in[0, T)$, where $Y_{t}^{\varepsilon}$ is the solution of BSDE (2.1). Moreover, if $g(\omega, \cdot, y, z)$ is continuous, the latter equality holds for all $t \in[0, T)$.

\section{Probabilistic interpretation of $\mathrm{HJB}$ equations}

In this section we will show the probabilistic interpretation for a generalized HJB equations, in the viscosity sense, which are associated with stochastic recursive optimal control problems. Before that, we should give a DPP for a stochastic recursive optimal control problem of the cost functional described by a controlled FBSDE system. This DPP is a well-known result and can be obtained by corresponding results in Peng [20] and $\mathrm{Pu}$ and Zhang [21], so we omit its proof.

The set $\mathcal{U}$ of admissible control processes is defined by

$$
\mathcal{U}:=\left\{\left(v_{t}\right)_{t \in[0, T]}: v(\cdot) \in \mathcal{H}^{2}\left(0, T ; \mathbf{R}^{k}\right) \text { and takes values in a compact set } U \subset \mathbf{R}^{k}\right\} .
$$

For a given admissible control $v(\cdot) \in \mathcal{U}$, we consider the following FBSDE system:

$$
\left\{\begin{array}{l}
X_{s}^{t, x ; v}=x+\int_{t}^{s} b\left(r, X_{r}^{t, x ; v}, v_{r}\right) \mathrm{d} r+\int_{t}^{s} \sigma\left(r, X_{r}^{t, x ; v}, v_{r}\right) \mathrm{d} B_{r}, \\
Y_{s}^{t, x ; v}=\Phi\left(X_{T}^{t, x ; v}\right)+\int_{s}^{T} g\left(r, X_{r}^{t, x ; v}, Y_{r}^{t, x ; v}, Z_{r}^{t, x ; v}, v_{r}\right) \mathrm{d} r-\int_{s}^{T}\left\langle Z_{r}^{t, x ; v}, \mathrm{~d} B_{r}\right\rangle, s \in[t, T],
\end{array}\right.
$$

where $t \in[0, T]$ is the initial time, $x \in \mathbf{R}^{n}$ is the initial state, and mappings $b:[0, T] \times$ $\mathbf{R}^{n} \times U \mapsto \mathbf{R}^{n}, \sigma:[0, T] \times \mathbf{R}^{n} \times U \mapsto \mathbf{R}^{n \times d}, g:[0, T] \times \mathbf{R}^{n} \times \mathbf{R} \times \mathbf{R}^{d} \times U \mapsto \mathbf{R}, \Phi: \mathbf{R}^{n} \mapsto \mathbf{R}$ satisfy the following conditions: 
(H1) For each $x \in \mathbf{R}^{n}, y \in \mathbf{R}, z \in \mathbf{R}^{d}$ and $v \in U, b(\cdot, x, v), \sigma(\cdot, x, v)$ and $g(\cdot, x, y, z, v)$ are continuous;

(H2) There exists a constant $K \geq 0$ such that for each $x, x^{\prime} \in \mathbf{R}^{n}$, and $v, v^{\prime} \in U$,

$$
\left|b(t, x, v)-b\left(t, x^{\prime}, v^{\prime}\right)\right|+\left|\sigma(t, x, v)-\sigma\left(t, x^{\prime}, v^{\prime}\right)\right| \leq K\left(\left|x-x^{\prime}\right|+\left|v-v^{\prime}\right|\right)
$$

(H3) There exists a constant $K \geq 0$ such that for each $x, x^{\prime} \in \mathbf{R}^{n}, y, y^{\prime} \in \mathbf{R}, z, z^{\prime} \in \mathbf{R}^{d}$, $v, v^{\prime} \in U$

$$
\left|g(t, x, y, z, v)-g\left(t, x^{\prime}, y^{\prime}, z^{\prime}, v^{\prime}\right)\right|+\left|\Phi(x)-\Phi\left(x^{\prime}\right)\right| \leq K\left(\left|x-x^{\prime}\right|+\left|y-y^{\prime}\right|+\left|z-z^{\prime}\right|+\left|v-v^{\prime}\right|\right) .
$$

Obviously, under the above assumptions, for any $v(\cdot) \in \mathcal{U}$ the control system (3.1) admits a unique solution $\left(X^{t, x ; v}, Y^{t, x ; v}, Z^{t, x ; v}\right)$ in $\mathcal{S}^{2}\left(t, T ; \mathbf{R}^{n} \times \mathbf{R}\right) \times \mathcal{H}^{2}\left(t, T ; \mathbf{R}^{d}\right)$.

We now define the associated cost functional,

$$
J(t, x ; v(\cdot)):=\left.Y_{s}^{t, x ; v}\right|_{s=t}, \quad(t, x) \in[0, T] \times \mathbf{R}^{n}, v(\cdot) \in \mathcal{U},
$$

and define the value function of the stochastic recursive optimal control problem,

$$
u(t, x):=\operatorname{esssup}_{v(\cdot) \in \mathcal{U}} J(t, x ; v(\cdot)), \quad(t, x) \in[0, T] \times \mathbf{R}^{n} .
$$

Here by standard estimates for FBSDE (3.1) we know that $u(t, x)$ is well defined. Moreover, $u(t, x)$ is deterministic, continuous in $(t, x)$ and of at most linear growth with respect to $x$, see Peng [20] or Pu and Zhang [21] for a survey. To introduce the DPP, we need the notion of backward semigroups, which is originaly due to Peng [20]. For each $(t, x) \in[0, T] \times \mathbf{R}^{n}, v(\cdot) \in \mathcal{U}, 0 \leq \delta \leq T-t$ and a random variable $\xi \in L^{2}\left(\Omega, \mathcal{F}_{t+\delta}, \mathbf{P} ; \mathbf{R}\right)$, we denote $G_{t, t+\delta}^{t, x}[\xi]:=Y_{t}$, where $\left(Y_{s}, Z_{s}\right)_{s \in[t, t+\delta]}$ is the solution of the following BSDE,

$$
Y_{s}=\xi+\int_{s}^{t+\delta} g\left(r, X_{r}^{t, x ; v}, Y_{r}, Z_{r}, v_{r}\right) \mathrm{d} r-\int_{s}^{t+\delta}\left\langle Z_{r}, \mathrm{~d} B_{r}\right\rangle, \quad s \in[t, t+\delta] .
$$

Then for the control system (3.1) we have that $G_{t, T}^{t, x ; v}\left[\Phi\left(X_{T}^{t, x ; v}\right)\right]=G_{t, t+\delta}^{t, x ; v}\left[Y_{t+\delta}^{t, x ; v}\right]$.

Theorem 3.1 (DPP). Assume that (H1)-(H3) hold. Then the value function $u(t, x)$ enjoys the following dynamic programming principle, for each $0 \leq \delta \leq T-t$,

$$
u(t, x)=\sup _{v(\cdot) \in \mathcal{U}} G_{t, t+\delta}^{t, x ; v}\left[u\left(t+\delta, X_{t+\delta}^{t, x ; v}\right)\right]
$$

Next we will relate the value function (3.2) with the following generalized HJB equation, which is a fully nonlinear second order PDE of parabolic type,

$$
\left\{\begin{array}{l}
\partial_{t} u(t, x)+\sup _{v \in U}\left\{\mathcal{L}_{t}^{v} u(t, x)+g\left(t, x, u(t, x), \sigma^{*}(t, x, v) \nabla u(t, x), v\right)\right\}=0 \\
u(T, x)=\Phi(x)
\end{array}\right.
$$

where $\mathcal{L}_{t}^{v}$ is a family of second order partial differential operators,

$$
\mathcal{L}_{t}^{v} u:=\frac{1}{2} \operatorname{Tr}\left\{\sigma \sigma^{*}(t, x, v) D^{2} u\right\}+\langle b(t, x, v), \nabla u\rangle .
$$

We would like to prove that the value function $u(t, x)$ defined in (3.2) is a viscosity solution of the HJB equation (3.3). We first recall the notion of viscosity solution for (3.3), which is adapted from Crandall, Ishii and Lions [6] and Peng [20]. 
Definition 3.2. A function $u \in C\left([0, T] \times \mathbf{R}^{n} ; \mathbf{R}\right)$ is called a viscosity subsolution (resp., supersolution) of the HJB equation (3.3), if $u(T, x) \leq \Phi(x)$ (resp., $u(T, x) \geq \Phi(x)$ ) for all $x \in \mathbf{R}^{n}$, and for any $\varphi \in C_{b}^{1,2}\left([0, T] \times \mathbf{R}^{n} ; \mathbf{R}\right)$ and $(t, x) \in[0, T) \times \mathbf{R}^{n}$ is a local minimum (resp., maximum) point of $\varphi-u$, then

$$
\left.\partial_{t} \varphi(t, x)+\sup _{v \in U}\left\{\mathcal{L}_{t}^{v} \varphi(t, x)+g\left(t, x, u(t, x), \sigma^{*}(t, x, v) \nabla \varphi(t, x), v\right)\right\} \geq \text { (resp., } \leq\right) 0 .
$$

A function $u \in C\left([0, T] \times \mathbf{R}^{n} ; \mathbf{R}\right)$ is called a viscosity solution of (3.3) if it is both a viscosity subsolution and a viscosity supersolution.

Theorem 3.3 (Probabilistic interpretation). Let assumptions (H1)-(H3) hold. Then the value function $u(t, x)$ defined by (3.2) is a viscosity solution of the HJB equation (3.3).

Proof. Note that $u(t, x)$ is continuous in $(t, x)$. We first prove that $u$ is a viscosity supersolution. Take any $\varphi \in C_{b}^{1,2}\left([0, T] \times \mathbf{R}^{n} ; \mathbf{R}\right),(t, x) \in[0, T) \times \mathbf{R}^{n}$ such that $\varphi-u$ achieves the local maximum at $(t, x)$. Without loss of generality, we assume $u(t, x)=$ $\varphi(t, x)$. Since $u(T, x)=\Phi(x)$ holds for all $x \in \mathbf{R}^{n}$, it reduces to prove that

$$
\partial_{t} \varphi(t, x)+\sup _{v \in U}\left\{\mathcal{L}_{t}^{v} \varphi(t, x)+g\left(t, x, u(t, x), \sigma^{*}(t, x, v) \nabla \varphi(t, x), v\right)\right\} \leq 0 .
$$

It follows from Theorem 3.1 that for each $0<\delta \leq T-t$,

$$
\varphi(t, x)=u(t, x)=\sup _{v(\cdot) \in \mathcal{U}} G_{t, t+\delta}^{t, x ; v}\left[u\left(t+\delta, X_{t+\delta}^{t, x ; v}\right)\right] .
$$

The fact that $\varphi \leq u$ and the monotonicity of backward semigroup $G$ (or the comparison theorem for solutions of BSDEs, Theorem 2.2 in El Karoui, Peng and Quenez [7]) yield that

$$
\sup _{v(\cdot) \in \mathcal{U}}\left\{G_{t, t+\delta}^{t, x ; v}\left[\varphi\left(t+\delta, X_{t+\delta}^{t, x ; v}\right)\right]-\varphi(t, x)\right\} \leq 0 .
$$

For each $v(\cdot) \in \mathcal{U}$, we set $Y_{t}^{v, \delta}:=G_{t, t+\delta}^{t, x ; v}\left[\varphi\left(t+\delta, X_{t+\delta}^{t, x ; v}\right)\right]$, which is a solution of the following BSDE,

$$
Y_{t}^{v, \delta}=\varphi\left(t+\delta, X_{t+\delta}^{t, x ; v}\right)+\int_{t}^{t+\delta} g\left(r, X_{r}^{t, x ; v}, Y_{r}^{v, \delta}, Z_{r}^{v, \delta}, v_{r}\right) \mathrm{d} r-\int_{t}^{t+\delta}\left\langle Z_{r}^{v, \delta}, \mathrm{d} B_{r}\right\rangle .
$$

Itô's formula to $\varphi\left(r, X_{r}^{t, x ; v}\right)$ on the time interval $[t, t+\delta]$ yields that

$$
\begin{aligned}
\varphi(t, x)= & \varphi\left(t+\delta, X_{t+\delta}^{t, x ; v}\right)-\int_{t}^{t+\delta}\left[\partial_{r} \varphi\left(r, X_{r}^{t, x ; v}\right)+\mathcal{L}_{r}^{v} \varphi\left(r, X_{r}^{t, x ; v}\right)\right] \mathrm{d} r \\
& -\int_{t}^{t+\delta}\left\langle\sigma^{*}\left(r, X_{r}^{t, x ; v}, v_{r}\right) \nabla \varphi\left(r, X_{r}^{t, x ; v}\right), \mathrm{d} B_{r}\right\rangle .
\end{aligned}
$$

Setting $\hat{Y}^{v, \delta}:=Y^{v, \delta}-\varphi\left(\cdot, X^{t, x ; v}\right), \hat{Z}^{v, \delta}:=Z^{v, \delta}-\sigma^{*}\left(\cdot, X^{t, x ; v}, v.\right) \nabla \varphi\left(\cdot, X^{t, x ; v}\right)$, we deduce that,

$$
\hat{Y}_{t}^{v, \delta}=\int_{t}^{t+\delta} F\left(r, X_{r}^{t, x ; v}, \hat{Y}_{r}^{v, \delta}, \hat{Z}_{r}^{v, \delta}, v_{r}\right) \mathrm{d} r-\int_{t}^{t+\delta}\left\langle\hat{Z}_{r}^{v, \delta}, \mathrm{d} B_{r}\right\rangle
$$

where for each $r \in[t, t+\delta], x \in \mathbf{R}^{n}, y \in \mathbf{R}, z \in \mathbf{R}^{d}$ and $v \in U$,

$$
F(r, x, y, z, v):=\partial_{r} \varphi(r, x)+\mathcal{L}_{r}^{v} \varphi(r, x)+g\left(r, x, y+\varphi(r, x), z+\sigma^{*}(r, x, v) \nabla \varphi(r, x), v\right) .
$$

Now by (3.5) we have that for each $0<\delta \leq T-t, \sup _{v(\cdot) \in \mathcal{U}} \hat{Y}_{t}^{v, \delta} \leq 0$. Hence, we deduce that $\hat{Y}_{t}^{v^{\prime}, \delta} \leq 0$ holds for each $v^{\prime} \in U$. It is obvious that (A1) and (A2) hold true for 
$F\left(r, X_{r}^{t, x ; v}, y, z, v_{r}\right)$ since $g$ satisfies (H1) and (H3). Then (2.3) in Theorem 2.1 implies that for each $v^{\prime} \in U$,

$$
F\left(t, x, 0,0, v^{\prime}\right)=\lim _{\delta \rightarrow 0} \frac{\hat{Y}_{t}^{v^{\prime}, \delta}}{\delta} \leq 0 .
$$

Thereby, we know that (3.4) holds by the definition of $F$.

Finally, we prove that $u$ is a viscosity subsolution. Take any $\varphi \in C_{b}^{1,2}\left([0, T] \times \mathbf{R}^{n} ; \mathbf{R}\right)$ such that $\varphi-u$ achieves the local minimum 0 at $(t, x) \in[0, T) \times \mathbf{R}^{n}$. We only need to prove that

$$
\partial_{t} \varphi(t, x)+\sup _{v \in U}\left\{\mathcal{L}_{t}^{v} \varphi(t, x)+g\left(t, x, u(t, x), \sigma^{*}(t, x, v) \nabla \varphi(t, x), v\right)\right\} \geq 0 .
$$

In this case we have $\varphi \geq u$, then the monotonicity of back semigroup $G$ leads to that for each $0<\delta \leq T-t$,

$$
\sup _{v(\cdot) \in \mathcal{U}}\left\{G_{t, t+\delta}^{t, x ; v}\left[\varphi\left(t+\delta, X_{t+\delta}^{t, x ; v}\right)\right]-\varphi(t, x)\right\} \geq 0
$$

Note that the BSDE (3.6) still holds since its derivation relies only on the definition of back semigroups and Itô's formula. Then by the definitions of $Y_{t}^{v, \delta}$ and $\hat{Y}^{v, \delta}$ we can rewrite the inequality (3.8) as $\sup _{v(\cdot) \in \mathcal{U}} \hat{Y}_{t}^{v, \delta} \geq 0$. Thus, there exists a sequence $\left\{v^{i}(\cdot)\right\}_{i \geq 1} \subset \mathcal{U}$ such that, $\mathbf{P}-$ a.s., $\sup _{v(\cdot) \in \mathcal{U}} \hat{Y}_{t}^{v, \delta}=\sup _{i \geq 1} \hat{Y}_{t}^{v^{i}, \delta}$. For each $0<\delta \leq T-t$ and $\varepsilon>0$, we define

$$
\widetilde{\Gamma}_{i}:=\left\{\sup _{v(\cdot) \in \mathcal{U}} \hat{Y}_{t}^{v, \delta} \leq \hat{Y}_{t}^{v^{i}, \delta}+\delta \varepsilon\right\} \in \mathcal{F}_{t}, \quad i \geq 1 .
$$

Then the events $\Gamma_{1}:=\widetilde{\Gamma}_{1}, \Gamma_{i}:=\widetilde{\Gamma}_{i} /\left(\cup_{j=1}^{i-1} \widetilde{\Gamma}_{j}\right) \in \mathcal{F}_{t}, i \geq 2$ are mutually disjoint and form a $\left(\Omega, \mathcal{F}_{t}\right)$-partition. Obviously, we have that $v^{\varepsilon}(\cdot):=\sum_{i>1} \mathbf{1}_{\Gamma_{i}} v^{i}(\cdot) \in \mathcal{U}$. And from the uniqueness for solutions of BSDEs, we know that $\mathbf{P}-$ a.s., $\hat{Y}_{t}^{v^{\varepsilon}, \delta}=\sum_{i \geq 1} \mathbf{1}_{\Gamma_{i}} \hat{Y}_{t}^{v^{i}, \delta}$. Hence, we conclude that, $\mathbf{P}-a . s$.,

$$
\hat{Y}_{t}^{v^{\varepsilon}, \delta}=\sum_{i \geq 1} \mathbf{1}_{\Gamma_{i}} \hat{Y}_{t}^{v^{i}, \delta} \geq \sup _{v(\cdot) \in \mathcal{U}} \hat{Y}_{t}^{v, \delta}-\delta \varepsilon \geq-\delta \varepsilon .
$$

We now suppose that (3.7) does not hold. Then by the definition of $F$, there exists a $\varepsilon_{0}>0$ such that $F(t, x, 0,0, v)<-\varepsilon_{0}$ for each $v \in U$. Since $F(\cdot, x, 0,0, \cdot)$ is uniformly continuous, when $\delta$ is small enough we derive that $F(r, x, 0,0, v)<-\varepsilon_{0} / 2$ for each $r \in[t, t+\delta]$ and $v \in U$. Applying (2.2) in Theorem 2.1 we have that for each $t \in[0, T]$,

$$
\lim _{\delta \rightarrow 0} \frac{1}{\delta} \mathbf{E}\left[\left|\hat{Y}_{t}^{v^{\varepsilon}, \delta}-\int_{t}^{t+\delta} F\left(r, X_{r}^{t, x ; v^{\varepsilon}}, 0,0, v_{r}^{\varepsilon}\right) \mathrm{d} r\right|\right]=0 .
$$

Then, for $\varepsilon_{0} / 4>0$, there exists a small enough $\delta>0$ such that

$$
\frac{1}{\delta} \mathbf{E}\left[\hat{Y}_{t}^{v^{\varepsilon}, \delta}\right] \leq \frac{1}{\delta} \mathbf{E}\left[\int_{t}^{t+\delta} F\left(r, X_{r}^{t, x ; v^{\varepsilon}}, 0,0, v_{r}^{\varepsilon}\right) \mathrm{d} r\right]+\frac{\varepsilon_{0}}{4} .
$$

Noticing that $\hat{Y}_{t}^{v^{\varepsilon}, \delta} \geq-\delta \varepsilon$ and the following two estimates, where $C \geq 0$ and $p \geq 1$ are two constants,

$$
\begin{gathered}
\left|F\left(r, X_{r}^{t, x ; v^{\varepsilon}}, 0,0, v_{r}^{\varepsilon}\right)-F\left(r, x, 0,0, v_{r}^{\varepsilon}\right)\right| \leq C\left(1+|x|^{2}\right)\left(\left|X_{r}^{t, x ; v^{\varepsilon}}-x\right|+\left|X_{r}^{t, x ; v^{\varepsilon}}-x\right|^{3}\right), \\
\mathbf{E}\left[\sup _{r \in[t, t+\delta]}\left|X_{r}^{t, x ; v^{\varepsilon}}-x\right|^{p}\right] \leq C \delta^{p / 2}
\end{gathered}
$$


we can take $\delta \rightarrow 0$ and then combine with $\mathbf{P}-$ a.s., $F\left(r, x, 0,0, v_{r}^{\varepsilon}\right)<-\varepsilon_{0} / 2$, obtaining that

$$
-\varepsilon \leq \lim _{\delta \rightarrow 0} \frac{1}{\delta} \mathbf{E}\left[\int_{t}^{t+\delta} F\left(r, x, 0,0, v_{r}^{\varepsilon}\right) \mathrm{d} r\right]+\frac{\varepsilon_{0}}{4} \leq-\frac{\varepsilon_{0}}{2}+\frac{\varepsilon_{0}}{4}=-\frac{\varepsilon_{0}}{4} .
$$

Hence, when $\varepsilon=\varepsilon_{0} / 6$ it will contradict with $\varepsilon_{0}>0$. So the inequality (3.7) holds. Therefore, the proof of Theorem 3.3 is finished.

Remark 3.4. From the proof procedure of Theorem 3.3, we can observe that if the DPP holds true, the probabilistic interpretation of HJB equations can be proved as soon as the representation theorem for generators of BSDE (3.6) holds true, where such theorem is determined by the conditions for the generator $g$ of the BSDE in (3.1). So our method also applies to the cases of Buckdahn and Li [4] and Pu and Zhang [21]. Moreover, the additional assumption, adopted in $\mathrm{Pu}$ and Zhang [21], that $g(t, x, y, z)$ is independent of $z$ can be naturally eliminated by the representation theorem in Fan, Jiang and Xu [8].

Next we introduce a space of continuous functions,

$$
\begin{aligned}
& \Theta:=\left\{\varphi \in C\left([0, T] \times \mathbf{R}^{n} ; \mathbf{R}\right): \text { There exists a constant } A>0\right. \text { such that } \\
&\left.\lim _{|x| \rightarrow \infty} \varphi(t, x) \exp \left\{-A\left(\log \sqrt{1+|x|^{2}}\right)^{2}\right\}=0, \text { uniformly in } t \in[0, T]\right\} .
\end{aligned}
$$

The growth condition of the functions in $\Theta$ is slightly weaker than the polynomial growth. Note that $u(t, x)$ defined in (3.2) belongs to $\Theta$ since it is of at most linear growth with respect to $x$. We give a comparison theorem, in the space $\Theta$, for viscosity subsolutions and supersolutions of the HJB equation (3.3), which yields that $u(t, x)$ is the uniqueness viscosity solution of (3.3). Since the proof is analogous to Theorem 5.3 of Buckdahn and Li [4], we just provide a sketch of this proof.

Theorem 3.5 (Comparison principle). Assume that (H1)-(H3) hold. Let $u_{1} \in \Theta$ and $u_{2} \in \Theta$ be, respectively, a viscosity subsolution and supersolution of (3.3). Then we have $u_{1}(t, x) \leq u_{2}(t, x)$ for each $(t, x) \in[0, T] \times \mathbf{R}^{n}$.

Proof. Suppose that the assumptions are in force, and define $w(t, x):=u_{1}(t, x)-u_{2}(t, x)$, $(t, x) \in[0, T] \times \mathbf{R}^{n}$. Then we have the following lemma.

Lemma 3.6. The function $w(t, x)$ is a viscosity subsolution of the following equation,

$$
\left\{\begin{aligned}
& \partial_{t} w(t, x)+\sup _{v \in U}\left\{\mathcal{L}_{t}^{v} w(t, x)+K|w(t, x)|\right. \\
&+K|\nabla w(t, x)||\sigma(t, x, v)|\}=0,(t, x) \in[0, T) \times \mathbf{R}^{n}, \\
& w(T, x)=0, \quad x \in \mathbf{R}^{n} .
\end{aligned}\right.
$$

The proof of this lemma is analogous to that of Lemma 3.7 in Barles, Buckdahn and Pardoux [1], so we omit it here. Next it is sufficient to prove that $w(t, x) \leq 0$. For any $A>0$, we define the function $\chi(t, x):=\exp \left\{\left(C_{1}(T-t)+A\right) \psi(x)\right\},(t, x) \in[0, T] \times \mathbf{R}^{n}$, where $\psi(x):=\left(\log \sqrt{1+|x|^{2}}+1\right)^{2}$ and the constant $C_{1}>0$ will be chosen later. By some direct computations we have the following properties,

$$
|\nabla \psi(x)| \leq \frac{2 \sqrt{\psi(x)}}{\sqrt{1+|x|^{2}}} \leq 4, \quad\left|D^{2} \psi(x)\right| \leq \frac{C(1+\sqrt{\psi(x)})}{1+|x|^{2}}, \quad x \in \mathbf{R}^{n},
$$

where and hereafter $C>0$ is a constant and may vary line by line. We denote $t_{1}:=$ $T-A / C_{1}$. Then for each $t \in\left[t_{1}, T\right]$, we have that

$$
\partial_{t} \chi(t, x)=-C_{1} \psi(x) \chi(t, x),|\nabla \chi(t, x)| \leq C \chi(t, x) \frac{\sqrt{\psi(x)}}{\sqrt{1+|x|^{2}}},\left|D^{2} \chi(t, x)\right| \leq C \chi(t, x) \frac{\psi(x)}{1+|x|^{2}} .
$$


Note that both $b$ and $\sigma$ are of linear growth with respect to $x$. By virtue with the previous estimates we can obtain that for each $(t, x) \in\left[t_{1}, T\right] \times \mathbf{R}^{n}$,

$$
\begin{aligned}
& \partial_{t} \chi(t, x)+\sup _{v \in U}\left\{\mathcal{L}_{t}^{v} \chi(t, x)+K|\chi(t, x)|+K|\nabla \chi(t, x)||\sigma(t, x, v)|\right\} \\
& \leq-\chi(t, x)\left[C_{1}-(2 C+K)\right] \psi(x)<0,
\end{aligned}
$$

provided that $C_{1}>2 C+K$.

Since both $u_{1}$ and $u_{2}$ belong to $\Theta$, we have that for some $A>0$,

$$
\lim _{|x| \rightarrow \infty} w(t, x) \exp \left\{-A\left(\log \sqrt{1+|x|^{2}}\right)^{2}\right\}=0,
$$

uniformly with respect to $t \in[0, T]$. Thus, for any $\varepsilon>0, w(t, x)-\varepsilon \chi(t, x)$ is bounded from above in $\left[t_{1}, T\right] \times \mathbf{R}^{n}$ and

$$
M(\varepsilon):=\max _{\left[t_{1}, T\right] \times \mathbf{R}^{n}}(w-\varepsilon \chi)(t, x) \exp \{-K(T-t)\}
$$

is achieved at some point $\left(t^{\varepsilon}, x^{\varepsilon}\right) \in\left[t_{1}, T\right] \times \mathbf{R}^{n}$. For each $(t, x) \in\left[t_{1}, T\right] \times \mathbf{R}^{n}$, define $\varphi(t, x):=\varepsilon \chi(t, x)+M(\varepsilon) \exp \{K(T-t)\}$. Then $\varphi \in C\left(\left[t_{1}, T\right] \times \mathbf{R}^{n} ; \mathbf{R}\right), \varphi\left(t^{\varepsilon}, x^{\varepsilon}\right)=w\left(t^{\varepsilon}, x^{\varepsilon}\right)$ and $w(t, x)-\varphi(t, x) \leq 0$ for each $(t, x) \in\left[t_{1}, T\right] \times \mathbf{R}^{n}$. We suppose that $M(\varepsilon)>0$ for some $\varepsilon>0$. Then $w\left(t^{\varepsilon}, x^{\varepsilon}\right)=\varphi\left(t^{\varepsilon}, x^{\varepsilon}\right)>0$, and by the definition of viscosity subsolution we have that

$$
\partial_{t} \varphi\left(t^{\varepsilon}, x^{\varepsilon}\right)+\sup _{v \in U}\left\{\mathcal{L}_{t}^{v} \varphi\left(t^{\varepsilon}, x^{\varepsilon}\right)+K\left|w\left(t^{\varepsilon}, x^{\varepsilon}\right)\right|+K\left|\nabla \varphi\left(t^{\varepsilon}, x^{\varepsilon}\right)\right|\left|\sigma\left(t^{\varepsilon}, x^{\varepsilon}, v\right)\right|\right\} \geq 0 .
$$

On the other hand, by some easy computations and (3.10) we deduce that

$$
\begin{aligned}
& \partial_{t} \varphi\left(t^{\varepsilon}, x^{\varepsilon}\right)+\sup _{v \in U}\left\{\mathcal{L}_{t}^{v} \varphi\left(t^{\varepsilon}, x^{\varepsilon}\right)+K\left|w\left(t^{\varepsilon}, x^{\varepsilon}\right)\right|+K\left|\nabla \varphi\left(t^{\varepsilon}, x^{\varepsilon}\right) \| \sigma\left(t^{\varepsilon}, x^{\varepsilon}, v\right)\right|\right\} \\
& =\varepsilon\left(\partial_{t} \chi\left(t^{\varepsilon}, x^{\varepsilon}\right)+\sup _{v \in U}\left\{\mathcal{L}_{t}^{v} \chi\left(t^{\varepsilon}, x^{\varepsilon}\right)+K\left|\chi\left(t^{\varepsilon}, x^{\varepsilon}\right)\right|+K\left|\nabla \chi\left(t^{\varepsilon}, x^{\varepsilon}\right)\right|\left|\sigma\left(t^{\varepsilon}, x^{\varepsilon}, v\right)\right|\right\}\right)<0 .
\end{aligned}
$$

This contradiction indicates that $M(\varepsilon) \leq 0$ holds for each $\varepsilon>0$. Thus we have that $w(t, x) \leq \varepsilon \chi(t, x)$ for all $t \in\left[t_{1}, T\right] \times \mathbf{R}^{n}$. Sending $\varepsilon \rightarrow 0$ yields that $w(t, x) \leq 0$. Finally, utilizing the same arguments in the intervals $\left[t_{i+1}, t_{i}\right](i=1,2, \cdots)$, where $t_{i+1}:=$ $\left(t_{i}-A / C_{1}\right)^{+}$, we can obtain that $w(t, x) \leq 0$ holds for each $(t, x) \in[0, T] \times \mathbf{R}^{n}$.

\section{Discussion}

Although this note concentrates on PDEs of HJB type, the representation theorem approach is applicable for the probabilistic interpretation of semilinear (or quasilinear) second order PDEs of parabolic types, see our previous works in Section 4 of Xiao and Fan [22]. Compared with the case of semilinear PDEs, the special representation form (2.2) in Theorem 2.1 is employed when proving viscosity subsolution, in order to treat the dependence on a control process $v(\cdot)$ of the coefficients. For semilinear PDEs, the representation theorem approach is easier since the functions $b, \sigma$ and $g$ are independent of the control process $v(\cdot)$.

There have been many attempts previously to study probabilistic interpretations in the viscosity sense by BSDEs after the initial works of Peng $[18,19]$. Yet existing methods are formidable to handle different situations in a unified way. As an alternative, researchers adopted different methods under different frameworks, such as strict comparison theorem for solutions of BSDEs under monotonicity condition and semilinear (quasilinear) PDEs (Pardoux, Pradeilles and Rao [15], Pardoux and Zhang [17], Pardoux 
[13], Pardoux and Tang [16]), approximation method for HJB or Isaacs equations (aforementioned Peng [20], Pu and Zhang [21], etc.). Usually, methods adopted for semilinear (or quasilinear) PDEs are not applicable to HJB equations.

Synthesizing the results of Section 4 in Xiao and Fan [22] and this note, the representation theorem method can be regarded as a feasible unified approach to the probabilistic interpretation of semilinear (quasilinear) and HJB type PDEs, whereas researchers can only focus on the representation theorem for generators of the corresponding BSDEs when proving the existence of viscosity solutions and ignore some redundant steps.

\section{References}

[1] Guy Barles, Rainer Buckdahn, and Etienne Pardoux, Backward stochastic differential equations and integral-partial differential equations, Stochastics Stochastics Rep. 60 (1997), no. $1-2,57-83$. MR-1436432

[2] Philippe Briand, François Coquet, Ying Hu, Jean Mémin, and Shige Peng, A converse comparison theorem for BSDEs and related properties of $g$-expectation, Electron. Comm. Probab. 5 (2000), no. 13, 101-117. MR-1781845

[3] Rainer Buckdahn and Ying Hu, Probabilistic interpretation of a coupled system of HamiltonJacobi-Bellman equations, J. Evol. Equ. 10 (2010), no. 3, 529-549. MR-2674058

[4] Rainer Buckdahn and Juan Li, Stochastic differential games and viscosity solutions of Hamilton-Jacobi-Bellman-Isaacs equations, SIAM J. Control Optim. 47 (2008), no. 1, 444-475. MR-2373477

[5] Rainer Buckdahn and Tianyang Nie, Generalized Hamilton-Jacobi-Bellman equations with Dirichlet boundary and stochastic exit time optimal control problem, SIAM J. Control Optim. 54 (2016), no. 2, 602-631. MR-3472008

[6] Michael G. Crandall, Hitoshi Ishii, and Pierre-Louis Lions, User's guide to viscosity solutions of second order partial differential equations, Bull. Amer. Math. Soc. (N.S.) 27 (1992), no. 1, 1-67. MR-1118699

[7] Nicole El Karoui, Shige Peng, and Marie Claire Quenez, Backward stochastic differential equations in finance, Math. Finance 7 (1997), no. 1, 1-71. MR-1434407

[8] Shengjun Fan, Long Jiang, and Yingying Xu, Representation theorem for generators of BSDES with monotonic and polynomial-growth generators in the space of processes, Electron. J. Probab. 16 (2011), no. 27, 830-844. MR-2793241

[9] Long Jiang, Representation theorems for generators of backward stochastic differential equations and their applications, Stochastic Process. Appl. 115 (2005), no. 12, 1883-1903. MR-2178500

[10] Long Jiang, Convexity, translation invariance and subadditivity for $g$-expectations and related risk measures, Ann. Appl. Probab. 18 (2008), no. 1, 245-258. MR-2380898

[11] Juan Li and Shanjian Tang, Optimal stochastic control with recursive cost functionals of stochastic differential systems reflected in a domain, ESAIM Control Optim. Calc. Var. 21 (2015), no. 4, 1150-1177. MR-3395759

[12] Juan Li and Qingmeng Wei, Optimal control problems of fully coupled FBSDEs and viscosity solutions of Hamilton-Jacobi-Bellman equations, SIAM J. Control Optim. 52 (2014), no. 3, 1622-1662. MR-3205734

[13] Etienne Pardoux, BSDEs, weak convergence and homogenization of semilinear PDEs, Nonlinear analysis, differential equations and control (F. H. Clarke, R. J. Stern, and G. Sabidussi, eds.), pp. 503-549, NATO Sci. Ser. C Math. Phys. Sci., vol. 528, Kluwer Acad. Publ., Dordrecht, 1999. MR-1695013

[14] Etienne Pardoux and Shige Peng, Adapted solution of a backward stochastic differential equation, Systems Control Lett. 14 (1990), no. 1, 55-61. MR-1037747

[15] Etienne Pardoux, Frédéric Pradeilles, and Zusheng Rao, Probabilistic interpretation of a system of semi-linear parabolic partial differential equations, Ann. Inst. H. Poincaré Probab. Statist. 33 (1997), no. 4, 467-490. MR-1465798 
[16] Etienne Pardoux and Shanjian Tang, Forward-backward stochastic differential equations and quasilinear parabolic PDEs, Probab. Theory Related Fields 114 (1999), no. 2, 123-150. MR-1701517

[17] Etienne Pardoux and Shuguang Zhang, Generalized BSDEs and nonlinear Neumann boundary value problems, Probab. Theory Related Fields 110 (1998), no. 4, 535-558. MR-1626963

[18] Shige Peng, Probabilistic interpretation for systems of quasilinear parabolic partial differential equations, Stochastics Stochastics Rep. 37 (1991), no. 1-2, 61-74. MR-1149116

[19] Shige Peng, A generalized dynamic programming principle and Hamilton-Jacobi-Bellman equation, Stochastics Stochastics Rep. 38 (1992), no. 2, 119-134. MR-1274898

[20] Shige Peng, Backward stochastic differential equations - stochastic optimization theory and viscosity solutions of HJB equations, Topics on stochastic analysis (In Chinese) (Jiaan Yan, Shige Peng, Shizan Fang, and Liming Wu, eds.), Science Press, Beijing, 1997, pp. 85-138.

[21] Jiangyan Pu and Qi Zhang, Dynamic programming principle for stochastic recursive control problem with non-Lipschitz aggregator and associated Hamilton-Jacobi-Bellman equation, ESAIM Control Optim. Calc. Var. 24 (2018), no. 1, 355-376. MR-3843188

[22] Lishun Xiao and Shengjun Fan, A representation theorem for generators of BSDEs with general growth generators in $y$ and its applications, Statist. Probab. Lett. 129 (2017), 297305. MR-3688547

Acknowledgments. The first author expresses his gratitude to Dr. Yu Zhuo (School of Mathematical Sciences, Fudan University) for her many helpful discussions that led to the improved version of this note. The authors would like to express great thanks to the anonymous referees for their careful works and thoughtful suggestions that also helped to improve this note substantially. 